\title{
What IS ENGINEERING SCIENCE? DEFINING A DisCIPLINE Through a Cross-Institutional CoMParison AND A Multi- INSTITUTIONAL WORKSHOP
}

\author{
Lisa Romkey, Nikita Dawe and Rubaina Khan \\ University of Toronto \\ lisa.romkey@utoronto.ca
}

\begin{abstract}
The Division of Engineering Science at the University of Toronto offers a complex, multidisciplinary undergraduate program, commonly known as "EngSci". We are in the first of a multi-year project titled ROLE (Realigning Outcomes with Learning Experiences), designed to proactively realign curriculum, pedagogy, students, and brand with our program goals. The first step in this process is to understand the state of Engineering Science as an academic discipline more broadly, and to better understand its role in the broader engineering and science landscape.
\end{abstract}

To better understand the discipline, we have used the academic plan model to compare eight engineering science programs from around the globe. The academic plan model supports the identification of internal and external factors that shape academic programs and frames the academic plan itself as seven related components that make up curriculum. Utilizing public-facing documentation such as websites and grey literature, we compared the IESC (International Engineering Science Consortium) programs and found differences in fundamental curriculum content, sub-disciplinary foci, organizational structure, and sources of external influence.

Concurrently, we conducted a workshop with members from the IESC to facilitate dialogue on the state of the discipline. This workshop resulted in a number of interesting artifacts, documenting the perspective of the participants. Some key themes that emerged included a strong focus on fundamentals and first principles; a focus on non-traditional and rapidly developing sub-disciplines, using the notion that Engineering Science can act as an "incubator" for new disciplines; and a diversity of views on the relationship between science and engineering within Engineering Science programs.

Finally, the paper paves a way forward for the next phase of the work, which involves interviewing program faculty and alumni to further understand perceptions of the discipline and the positioning of the discipline in the broader science and engineering landscape.

Keywords: Engineering Science, Academic Disciplines, Curriculum, Higher Education

\section{INTRODUCTION}

The Division of Engineering Science at the University of Toronto offers a complex, multidisciplinary undergraduate program, commonly known as "EngSci". Engineering Science is a discipline with a rich history, established to better integrate science and engineering, and to address perceived gaps that resulted from an emphasis on the practical training of engineers; in the 1930's, leadership in engineering education at the University of Toronto made specific efforts to separate engineering from science, and focus on the practical training of professional engineers without instruction in the pure sciences. Engineering Science was initially "Engineering Physics", but later became "Engineering Science" when chemical engineering was offered as an area of specialization. Today, the "Science" in EngSci represents the program's more general foundation in science and mathematics.

In response to more recent changes in engineering practice and education, and technological development more broadly, EngSci now includes a number of multidisciplinary majors, and our students are showing a growing interest in computational science, machine learning, and entrepreneurial opportunities. While the International Engineering Science Consortium (IESC), of which EngSci is a member, describes the discipline of Engineering Science as "An interdisciplinary field bridging the gap between scientific theory and engineering applications with emphasis on the integration of mathematical, scientific, engineering and arts principles"[1], our program has evolved to include other characteristics. However, these have not been examined in any kind of a rigourous way - from determining how people think about the program, to how the characteristics 
of Engineering Science are actually promoted in the program.

We are in the first of a multi-year project titled ROLE (Realigning Outcomes with Learning Experiences), designed to proactively realign our curriculum, pedagogy, students, and brand with our program goals. More specifically, the project includes four main components: (i) the review and refinement of program goals; (ii) the redesign of several aspects of the program learning environment; (iii) finding new ways to support our students and faculty, and (iv) examining our brand strategy and admissions criteria.

The first step in the project is to understand the state of Engineering Science as an academic discipline, and to better understand its role in the broader engineering and science landscape. This work is the focus of this paper.. First, we examined the higher education literature on the construction and evolution of academic disciplines, using this as a lens for reconsidering the history and development of Engineering Science at the University of Toronto. Second, we conducted a comparison of the various Engineering Science programs that make up the Consortium. Finally, in June 2019, we held a workshop at the University of Toronto, as part of the biannual consortium meeting, to discuss the nature of Engineering Science. The results of this workshop were analyzed and are included in this paper.

\section{ENGINEERING SCIENCE - A DISCIPLINE?}

\subsection{Why disciplines exist}

Disciplines can be viewed as tools that shape how knowledge is mobilised and validated. They offer specific lenses through which the world can be observed, understood, and shaped. Donald [2] points out that "the method by which knowledge is arrived at in a discipline, the process of knowledge validation, and the truth criteria employed in that process are essential to the definition of a discipline" (p. 6). Disciplines find their place in universities through departments that members refer to as their "academic home". Poole [3] describes these departments as the managers of resources that are to be secured for its members to flourish. However, to function independently, disciplines need to identify their key defining features. Winchester [4] illustrated how intricate and complex the assignment of defining tenets for a discipline can be. A defining highlight, he stated, is that disciplines are both persistent yet changing through time; however, how these changes occur and are motivated vary by discipline. In general, disciplines can be perceived as perspectives for learning, each portrayed by specific perspectives, strategies, and practices that are representative of its community.

\subsection{What are the criteria of a discipline?}

Differences in disciplines can be seen through their activities, knowledge structures, social constructions, and communication patterns, which offers rich, complex, and rhetoric attributes that can be analysed and categorised. The boundaries of an academic discipline are defined by a common cognitive or social framework, which provides a logical way to differentiate within an organizational structure or organisation of learning that prioritizes the systematic production of new knowledge ([5], [6]).

There is more to disciplines than a subject taught in an academic setting. Krishnan [7] proposes a wide-ranging list of characteristics which include: 1) disciplines possess an object of research which could be shared with another discipline, 2) there is a large body of growing knowledge with regards to the object of research that is specific to the discipline, 3) disciplines organize their knowledge through theories and concepts, 4) disciplines describe their object of research in specialized technical language, 5) disciplines have developed their own research methods that meet their research requirements, 6) disciplines offer their corpus of knowledge through courses that are taught in the university or colleges. All disciplines do not comply with all of the characteristics above, however, a certain field of inquiry becomes recognised when the discipline can reproduce itself by producing a unique body of scholarship.

Trowler [8] contends that categorization based on epistemological essentialism overlooks the worthiness of considering the sociocultural dimension of higher education practices, including the costs of power dynamics and the development of biases within particular cultural contexts. Disciplinary cultures are theorized as open, natural organizations that are influenced by multi-causal relationships among participants, and impacted by external forces. Recognizing disciplines as cultures, "one recognizes that a field or subject-its knowledge, methods, theoretical approaches - cannot be separated from its practitioners" [9]. Trowler further purports that in addition to viewing disciplines to have an organizational character, the narratives constructed by the ones dwelling in the space is of importance: the impressions faculty have about other disciplines and subdisciplines are significant in creating a sort of reality themselves. Ylijoki [10] also agrees with Trowler by stating "besides the common cognitive basis, disciplines have their own social and cultural characteristics: norms, values, modes of interaction, lifestyle, pedagogical and ethical codes (p. 339). This focus on a discipline as a sociocultural entity is much more strongly aligned with Engineering Science, particularly given that from a knowledge generation and research perspective, the program draws from several established disciplines in the academy. 


\subsection{Considerations for Undergraduate EngSci as a Discipline}

Donald [11], in her book on learning within disciplines, examines the thinking processes employed in different disciplines and how they lead to the desired intellectual development of students within the discipline. First, she describes the learning environment each discipline offers. Second, she elaborates on the knowledge and higher-order thinking processes that are deemed important within a discipline and lastly, how these processes are cultivated in the student's learning and reasoning. The originality of the Engineering Science discipline lies in the teaching of math and sciences alongside fundamental engineering skills and knowledge. Sub- disciplines frequently change with a response to industry and research needs, and the program engages students in a multi-disciplinary experience. This structure has made the program agile as reflecting an active response to the global demand for technological changes, often creating the first undergraduate program in a new field. It has also, to some extent, defined the "thinking processes" in the program.

McArthur [12] points out that within a disciplinary discourse, new meanings formed are bounded by disciplinary assumptions within the setting of the social and cultural context. Hence, the path to constructing meaning of new knowledge does not follow a logical structured path, but through lived experiences of involvement in practices that invoke the generation of meaning-making such as "relevant discursive contexts, the taken-for-granted purposes, values and methods, the history of key debates, the influence of leading figures" ( $p$. 113). The Engineering Science programme, from its early years, emphasized one's ability to improve through metacognition and self-regulation; and provided students with a rigorous undergraduate curriculum. Focus on lifelong learning and a rigorous, first principles approach help define both the context and the discourse.

Engineering Science employs a number of features designed to build a community, such as staff-student meetings and room for students to convene and communicate. These socio-cultural aspects are not discipline-dependent, but rather the platforms each discipline offers for the trade of meaning within its discourses for its members. A fundamental method by which the communal members learn to see the same things when provoked with the same stimuli is through examples of situations that their predecessors in the community have already learned to see and deem them as accepted examples of knowledge [13]. This is particularly relevant to Engineering Science, given the large number of program alumni that are now instructors in the program. Hewson \& Hewson [14] note that the intellectual milieu in which a novice experiences its cultural beliefs, language, recognised theories, and facts and events, improves the development of certain concepts and impedes the development of others; for all of the reasons stated here, examining the Engineering Science community should be an important part of this research. Finally, the notion of a signature pedagogy - the specific instructional methodologies used in the preparation of members of a profession to develop particular "habits of mind" - is important to consider when examining Engineering Science [15] as a discipline. Our signature pedagogy might be a multi-disciplinary approach with curriculum integration; it might be learning through the development of a new discipline; it might be a first-principles approach to engineering problem solving. Through this project, we hope to define this and other defining features of our discipline.

\section{CROSS-INSTITUTIONAL COMPARISON}

\subsection{The Academic Plan Model}

We used Lattuca and Stark's Academic Plan Model [16] as a framework to compare Engineering Science programs in the International Engineering Science Consortium (IESC), as a way of furthering our understanding of Engineering Science as a discipline. The consortium was established in 2013, by five institutions, and now includes eight institutions with Engineering Science programs: University of California at Berkeley, KTH Royal Institute of Technology, Osaka University, National University of Singapore, University of Toronto, The University of Queensland, University College London and The University of Auckland. The consortium was developed to provide opportunities for academic and research exchange, and to share information between institutions in fields of mutual interest.

Appendix A is an illustration of the "academic plan in context". It is intended as a tool for curriculum development and revision at any level of detail from a single course module to a program or department. The "Academic Plan" itself reflects an integrated definition of curriculum that captures the range of decisions that instructors or curriculum developers make, and it is placed within the sociocultural context of influential factors within and outside of a university. The academic plan model supports the identification of internal and external factors that shape academic programs, allowing us to understand the organizational and socio-cultural contexts the different IESC programs operate within. With this understanding, we can analyse the curriculum structure of the programs according to the seven related components of the academic plan itself. The model reflects many of the themes described in Section 2.

Klassen and Donald [17] used the academic plan model to compare engineering leadership curricula in Canadian universities. Their focus was on specific courses and cocurricular programs whereas we recognized that some engineering science units in the consortium are entire schools with multiple departments, programs, and 
specialisations. It is not feasible at this stage to go into the same level of detail about curriculum content, sequence, and instruction when this differs between programs and courses within a given school; we instead make preliminary statements about the nature of these categories based on our overall understanding of engineering science at each university.

Our analysis focuses on undergraduate programs since these are more equivalent to our own Engineering Science program than graduate programs. We looked at material provided publicly through the consortium website and individual university websites to gather information about the programs. In our full analysis, we compared Engineering Science programs on the basis of the following:

- Sociocultural context: location, undergraduate unit, number of students, other undergraduate engineering programs offered, instructor home department, connection to graduate unit, accreditation and language

- Academic plan: program purpose, key content emphasis, subdisciplines, other curriculum features such as research and design opportunities, internship/co-op opportunities

In the discussion that follows, some of the key findings from this comparison of programs is presented.

\subsection{Analysis of Findings}

For elements of Lattuca and Stark's model, we include a simplified definition and identified similarities and differences between IESC institutions.

3.2.1 Internal influences. This includes organizational infrastructure and resources, instructor backgrounds, and the student body. Consortium members differ in student population, unit types, and whether they represent a standalone school or nested unit.

- $\quad$ Singapore and Berkeley have small (<300 students) programs within larger engineering units.

- Osaka and KTH have large standalone engineering science schools $(1000+$ students $)$ that are separate from other engineering and science schools within their institutions.

- Toronto has a similar number of students but is a division located within the larger engineering faculty.

- Auckland has a 450-student department within the engineering faculty.

- UCL classifies its entire engineering faculty (3300 students) as engineering sciences and has a separate Faculty of Mathematical and Physical Sciences

3.2.2 Instructor location(s): We wanted to identify whether instructors teaching in engineering science are dedicated faculty or are pulled in from their home units, especially if these home units are traditional science or engineering sub-disciplines. Based on our preliminary analysis, instructors at the standalone engineering science schools are housed within these schools, although they may work across faculties and programs within the schools. In the smaller engineering science programs and in the department/divisions, there may or may not be a group of dedicated instructors, while the rest are housed in other, mostly engineering departments. UCL draws attention to the fact that professors within a given engineering science department tend to have diverse backgrounds e.g. philosophers working in the civil engineering department.

Some universities' undergraduate engineering science units are connected to graduate engineering science units which do research and are distinct from their other engineering graduate units (Osaka, KTH, Auckland). Others, such as Toronto, have many of their engineering science undergraduates continue on to graduate programs in traditional engineering and science departments.

3.2.3 External influences. Due to our selection, all institutions are members of the IESC, though there is no evidence that consortium membership has had a direct influence in shaping the programs. Most programs are accredited by engineering bodies within their countries except for KTH's programs, which are accredited by the national education authority. Programs that are not accredited by engineering bodies include more interdisciplinary, scientific, and theoretical courses: Berkeley's program does not appear to be ABET accredited, and at UCL some programs are accredited by specific engineering bodies (e.g. Chemical Engineering is accredited by the Institution of Chemical Engineers) while others are not.

3.2.4 Purpose, content, and sequence. To assess how consortium members describe their curriculum to other members of the consortium, we extracted information about purposes and content from university profiles on the consortium website. In terms of program purpose, some universities focus on what engineering science graduates will do (solve complex or topical problems, develop new technology), others address where they might work (a wide range of fields, multidisciplinary areas), and some address how their programs facilitate the interplay between science and engineering. Four of the universities indicate that content emphasizes science, math, and engineering fundamentals, suggesting there is less focus on application or practical engineering work that is typically highlighted in traditional engineering disciplines. A more extensive analysis of the language used on university websites and within the academic calendars would provide a richer understanding of how the programs conceive of the purpose of engineering science as a discipline.

The sub-disciplines taught by the different universities as majors, programs, or specialisations also provide insight 
into the content of engineering science. It is challenging to group similar sub-disciplines without knowing more about them, for example "computer science," "computational engineering science," and "computer engineering" likely overlap to some extent, as would "engineering math and statistics," "mathematical science," "engineering mathematics," "engineering mathematics, statistics and finance," and "operations research." The most common sub-disciplines with consistent nomenclature include engineering physics (4), biomedical engineering (4), and energy or energy systems (3). Berkeley, NUS, Toronto, Auckland, and KTH offer sub-disciplines more closely associated with traditional and emerging engineering disciplines. Osaka includes sub-disciplines traditionally categorised as pure sciences e.g. chemistry. While UCL offers programs that cross the most disciplinary boundaries into business, medicine, and security/crime.

\section{IESC WORKSHOP}

Representatives from all eight members of the IESC came together in June 2019 for its biannual meeting. The biannual meeting is typically used to discuss items of common interest, such as research and student exchange. We took this opportunity to lead participants through an active workshop, designed to facilitate dialogue on the state of Engineering Science. Workshop participants were asked to define "engineering science", identify internal and external factors influencing their programs, and to consider content, instruction and learners to design elements of an academic plan for a hypothetical undergraduate engineering science program. This workshop resulted in a number of interesting artifacts, representing the perspective of the participants on both the current state of Engineering Science programs, as well as some of the aspirational goals related to the content, teaching practices and students in the program. Participants were placed in multi-institutional groups, to promote discussion comparing multiple institutions, and were asked to focus on program content, program learners and program instructors - and were given a number of specific prompts for each.

Workshop participants recorded discussion on worksheets, and we also recorded themes raised in small and full-group discussion. All of the data from these two sources was first organized by the major themes used in the workshop (learners, content, instruction, external forces and the definition of Engineering Science), and a number of themes were identified; some overlapping and some specific to each of the major themes. Then, a review of the data in these categories resulted in a reduction of themes to create a shorter list of over-arching themes that best reflect the data, and reflect the overlap between ideas in the major categories.

\subsection{Key Themes}

A number of key themes, representing how the participants conceptualize Engineering Science, emerged from the workshop. The responses in some cases represent perceptions of current practice, while in other cases, represent something more akin to the ideal.

4.1.1 Focus on Fundamentals and First Principles. Participants reference the value of using a first principles approach, to promote a deeper understanding of the underlying principles behind science and engineering, and to go one step "deeper" or "behind" other engineering programs. This can be accomplished with an emphasis on proofs, for example, rather than simply addressing the application of knowledge. Participants also described this as a "deductive" approach, with more fundamental math and science early in the program. There is a perception that students typically have the opportunity to participate in processes related to the generation of knowledge, for example through research opportunities.

Some participants suggesting moving "between theory and design, with each becoming more complex on each iteration". Another group provided a variation on that, suggesting that "the learning process - particularly in the upper years - includes exposure and reflection; projects cycle through doing and learning". One group suggested that "every course progresses from the general form to the specific (e.g. Navier-Stokes to Euler), so that the core pedagogy is "how to model" and not "which pre-existing model applies here".

4.1.2 Multi and Interdisciplinary Perspective. Through various discussions, participants focused on the interdisciplinary or multidisciplinary nature of Engineering Science. The role of interdisciplinary projects was prevalent in the discussions, with participants noting that these can "allow students to explore potential areas of specialization and facilitate iteration of specialization". Other discussions emphasized the role of multidisciplinary problems and design projects, and in particular that "realworld projects" necessitate an interdisciplinary or multidisciplinary approach; another group attached the importance of multi-disciplinary thinking to cutting edge fields, and finally another group noted that engineering science represents "the synergy of multifaceted approaches to the practice, research and design of engineering"; demonstrating that "multi" is not just strictly applied to the knowledge of disciplines, but also to the approaches and methods.

4.1.3 Integration. Following on the notion of inter and multi-disciplinarity is the notion of curriculum integration - through design projects, problem-based learning and the integration of courses more broadly. The participants provided a number of specific suggestions on integration, 
including: the addition of an "integration" course each term; specific modules that run between courses, a weeklong retreat for all instructors to integrate their material.

4.1.4 Innovation. Engineering Science was identified as a home for innovation, with the idea that Engineering Science focuses on non-traditional and rapidly developing sub-disciplines, acting as an "incubator". Participants identified that it was important to equip students with cutting edge tech, such as Machine Learning, but also that Engineering Science should promote the development of new disciplines and codes of practice in engineering. One group described Engineering Science as "a field that connects bleeding edge science/theory fields with bleeding edge engineering fields to produce science and engineering outcomes that otherwise could not be produced individually."

4.1.5 Unique Approaches to Problem Solving and Design. It's likely that any engineering education practitioners would speak about the relevance of engineering design and problem solving. In talking about Engineering Science specifically, there was a focus on "broad" challenges, "worlds biggest" problems with "no right answer", and acknowledgement of problems of a significant breadth in the scope of problems: from "pure science" to "human/sociological". Some participants noted that students should have to discern or identify the problem and not just solve it, which tends to prove more challenging. Finally, some participants focused on the notion of community - building a group of like-minded practitioners who explore multiple disciplines and a broad deep foundation together when solving problems; that we "solve problems (societal, technological or in fundamental science) by creating open-minded multidisciplinary communities".

\subsubsection{Relationship Between Engineering and Science.} There were various views on the relationship between science and engineering within Engineering Science programs. This relationship was described as a bridge; and by a second group, "Two-way bridging between cutting edge science and engineering". This group went on to articulate that "cutting edge science" elicits a science result; that "cutting edge engineering" elicits an engineering result, but that the bridge between the two elicits a "unique result"." Others included: engineering to enable to doing of science; using science to explain or perform engineering; and a combination or fusion of the two; for example, "Engineering Science is the fusion of science disciplines (that deals with creation of knowledge and understanding of nature) and the engineering disciplines (that deals with the application of knowledge to design/produce a useful product for mankind)." Participants also described Engineering Science as a more practical science, for example: "Takes ideas from science
\& makes them practical"; "Literally, engineering science - facilitating, directing, enabling science - an ACTIVE contributor to science"; and "Using science to perform or explain engineering".

\section{CONCLUSIONS AND NEXT STEPS}

This paper furthers the discussion on the characteristics that make and describe an academic discipline, offering a unique approach through the engineering lens. While there are few engineering science programs relative to traditional engineering disciplines, this research can help all engineering programs understand and define their disciplinary identities by teasing apart the differences between science, applied science, engineering science, and engineering disciplines, and by identifying the academic and sociocultural factors that define a discipline. We also demonstrate that Lattuca and Stark's academic plan model can serve as a useful framework for analyzing programs that differ in size and scope.

This paper also provides background for the next phase of the work which has recently commenced. This work involves interviewing several program faculty and alumni. There is a need for a shared understanding of Engineering Science to facilitate teaching innovation; research and educational collaborations; differentiation and promotion of programs, and to articulate the benefit of the program to society. These stakeholders will be asked about their perceptions of the program at the University of Toronto, views on the signature pedagogy for engineering science, and the positioning of the discipline in the broader science and engineering landscape. We will utilize the interviews to better understand the relevance of the programmatic themes described in section 4.1. The interviews will also provide an opportunity to further explore and situate the literature on academic disciplines described in section 2 .

The interviews are currently underway; 41 interview candidates have been identified and six interviews have already been completed. In addition to some of the themes described in section 4.1, these interview participants have discussed the program's reputation as challenging and attracting top students, how this "rigor" might be associated with over-assessment and related problems, unique curriculum elements such as senior thesis projects that reflect graduate-level research, and tensions as novel majors begin to overlap with streams offered in our Faculty's core engineering programs.

A further exploration and analysis of the consortium member programs will also be necessary to understand how certain program themes and choices come to be. For example, as described in section 3.2.4, different Engineering Science programs have different levels of "interdisciplinarity"; it's important to better understand the factors that have influenced this aspect of program design. Finally, both the interviews and the analysis of the consortium member programs will take a closer look at the 
sociocultural dimensions of Engineering Science; in other words, the social and cultural characteristics of the discipline, including norms, modes of interaction and pedagogical practices.

It is hoped that with a better understanding of what Engineering Science is - and what we want it to be - we can revise our program goals and learning outcomes, and then build curriculum and instructional strategies that are aligned with the goals, outcomes and overall mandate of the program. It is also critical that these goals and strategies acknowledge our socio-cultural context, and the many individuals who play a part in this complex undergraduate program.

\section{Acknowledgements}

We would like to acknowledge the Office of the Dean in the Faculty of Applied Science and Engineering, University of Toronto, for their generous support of our research. We would also like to thank the members of the International Engineering Science Consortium for their participation.

\section{References}

[1] "Outline," International Engineering Science Consortium. [Online]. Available: http://www.engineeringscience.org/outline/.

[2] J.G. Donald, "Disciplinary differences in knowledge validation", in Disciplinary differences in teaching and learning: Implications for practice, N. Hativa \& M. Marincovich, Eds. San Francisco, CA: Jossey-Bass, 1995, pp. 718.

[3] G. Poole, "Academic Disciplines: Homes or Barricades?", in The university and its disciplines: Teaching and learning within and beyond disciplinary boundaries, Carolin Kreber, Ed. New York: Routledge, 2008, pp. 50-57.

[4] I. Winchester, "On disciplines", Interchange, vol. 17, no. 2, pp. $178-185,1986$.

[5] T. Becher \& P.R. Trowler, "Academic tribes and territories: intellectual enquiry and the cultures of disciplines", Buckingham: Open University Press, 2001, 256 pp.

[6] M. Del Favero, “Academic disciplines", in Encyclopedia of education, J.W. Guthrie (Ed.), New York, NY: MacMillan,2003, pp. 9-14.

[7] A. Krishnan, "What are academic disciplines? Some observations on the disciplinarity vs. interdisciplinarity debate", 2009, available as of February 17, 2020 at http://eprints.ncrm.ac.uk/783/1/what are academic disciplines pdf.

[8] P. Trowler, "Beyond Epistemological Essentialism: Academic Tribes in the $21^{\text {st }}$ Century", in The university and its disciplines: Teaching and learning within and beyond disciplinary boundaries, Carolin Kreber, Ed. New York: Routledge, 2008, pp. 50-57.

[9] H.H. Bauer, "Barriers against interdisciplinarity: implications for studies of science, technology, and society", Science, Technology \& Human Values, vol. 15, no. 1, pp. 105119, 1990.

[10] O.H. Ylijoki, "Disciplinary Cultures and the Moral order of Studying”, Higher Education, vol. 39, pp. 339-362, 2000.

[11] J.G. Donald, Learning To Think: Disciplinary Perspectives. The Jossey-Bass Higher and Adult Education Series, JosseyBass: San Francisco, 2002.

[12] J. McArthur, "Diverse student voices within disciplinary discourses", in The university and its disciplines: teaching and learning within and beyond disciplinary boundaries, Carolin Kreber, Ed. New York: Routledge, 2009.

[13] T.S. Kuhn, The structure of scientific revolutions. Chicago: University of Chicago Press, 1970.

[14] P.W. Hewson \& M.G.B. Hewson, "The role of conceptual conflict in conceptual change and the design of science instruction", Instructional Science, vol. 13, no. 1, pp. 1-13.

[15] L. S. Shulman, "Signature Pedagogies in the Professions," Daedalus, vol. 134, no. 3, pp. 52-59, 2005.

[16] L. R. Lattuca and Joan S. Stark, "Curriculum: An Academic Plan," in Shaping the College Curriculum: Academic Plans in Context, San Francisco: Jossey-Bass, 2009, pp. 1-22.

[17] M. Klassen and J. Donald, "Using an Academic Plan Model to Analyze Canadian Engineering Leadership Curriculum," Proc. Can. Eng. Educ. Assoc., pp. 1-8, 2018. 
Appendix A: Academic Plan Model (Lattuca and Stark, 2009)

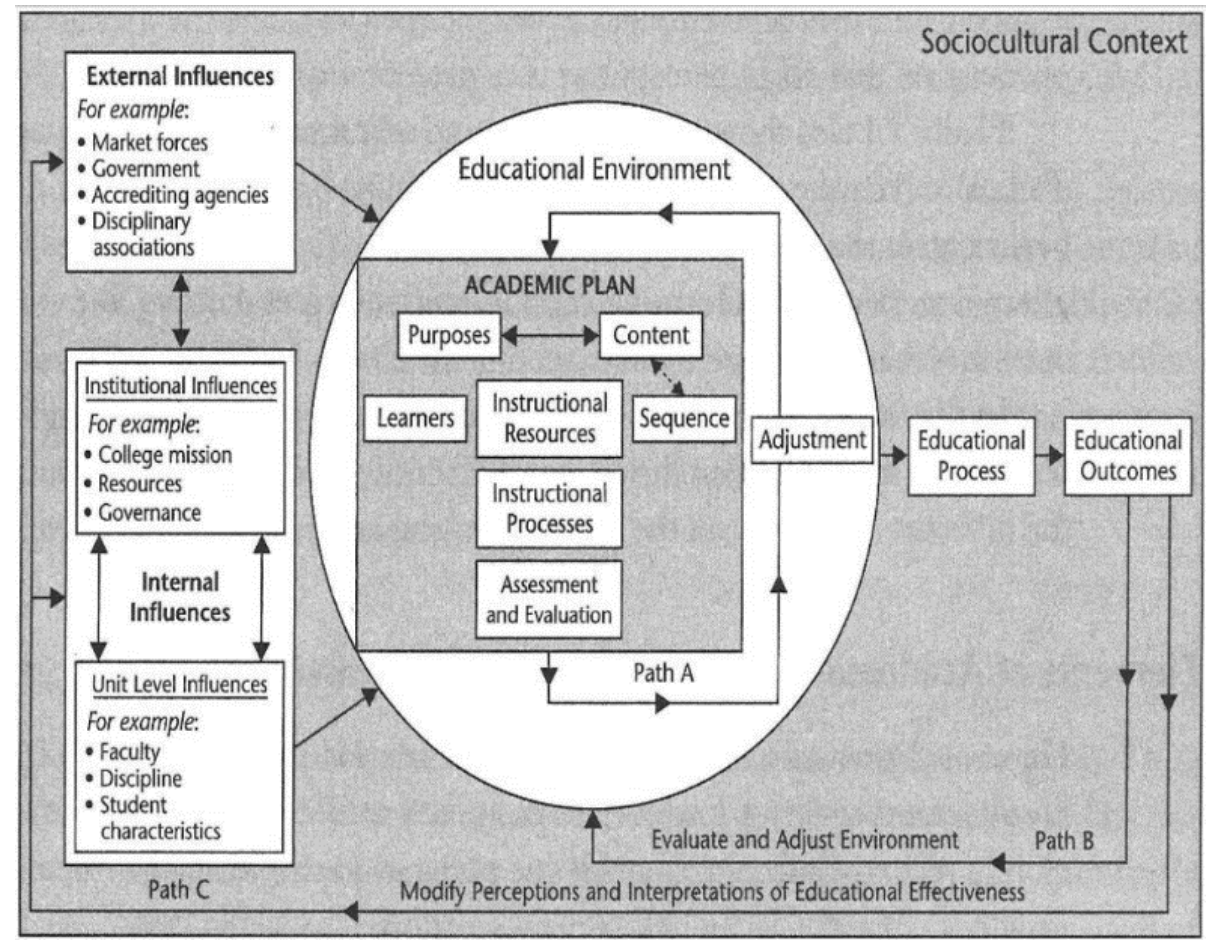

